\title{
It's now or later
}

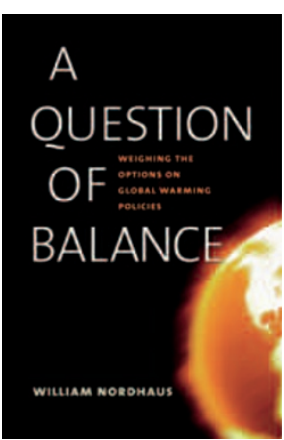

\section{A QUESTION OF BALANCE: WEIGHING THE OPTIONS ON GLOBAL WARMING POLICIES}

\author{
By William Nordhaus \\ Yale University Press: 2008. 256pp. US $\$ 28$

\section{Is a slow, measured approach to reducing emissions more cost-effective than taking immediate action?}

As the international debate about climate change moves from the question of whether climate change is taking place towards matters of how to deal with it, there remains much dispute about the costs of alternative policies. Among many environmentalists there is a presumption that almost all instruments intended to reduce emissions are desirable, but for fear of scaring politicians away from action there is a temptation to play down the costs. Yet costs will not go away, and William Nordhaus's latest contribution provides an excellent, accessible and compelling analysis of the main policy options, using the Dynamic Integrated model of Climate and the Economy

(DICE), which he has been developing for some years.

DICE integrates the science of climate change with estimates of its associated costs and damages into a single economic growth model. From this elegant and simple framework, Nordhaus provides a series of powerful and, for many, uncomfortable insights into climate policy. Among these, three stand out: the centrality of a price for carbon and estimates of its optimal level; the sensitivity of costs to the speed of emissions reductions - in particular, the very high costs of rapid near-term reductions, as proposed by economist Sir Nicholas Stern in his renowned 2006 review for the UK government, and variously by $\mathrm{Al}$ Gore; and the scale of the Kyoto framework's inefficiency.

Yet, interesting as the DICE framework is, none of these insights strictly requires DICE. The case for market-based instruments to establish a carbon price rests on much older and deeper economic arguments. The costs of setting too high a price for carbon and thereby accelerating emissions reductions in the face of a lumpy and fixed capital stock can be established independently of the DICE model. And it is not hard to appreciate that the Kyoto Protocol ignores cheaper carbon-reducing opportunities at the global level and encourages high-carbon industries to simply relocate outside of regions with binding caps. The advantage Nordhaus brings is to place numbers on these costs in terms of an implied price for carbon. The disadvantage, as Nordhaus is quick to recognize, is that these numbers may be interpreted as providing a spurious accuracy.

Where A Question of Balance has most power is where it is most controversial. Nordhaus tackles Stern head on. Stern's case for urgent action, which the DICE model shows would be excessively expensive in the short term, rests upon his radical assumption that the time discount rate should be close to zero. This means that we should value people's consumption equally regardless of whether they live now or in the future. Nordhaus has little time for this moral philosophy: he takes a much more positivistic position, grounded in market evidence and what people actually do, as reflected in market interest rates. The difference between Nordhaus's optimal climate change policy and Stern's policy based on a zero discount rate translates into a tenfold difference in the price of carbon. Stern's discounting approach, Nordhaus argues, gives too low a rate of return and too big a savings rate on climate-stabilizing investments compared with actual macroeconomic data. Not surprisingly, then, his verdict is damning.

Such a critique of Kyoto, and of current approaches to climate change policy, is valuable. Yet the book is itself open to two criticisms. First, on optimal policy, Nordhaus proposes a carbon tax or emissions trading to establish a carbon price. But he provides little guidance as to how this might be practically achieved and nothing on the political economy of its implementation. He is therefore open to the challenge that he is comparing actual policy against an ideal rather than against practical alternatives that take account of the political context. Second, Nordhaus is careful to recognize that his own results depend upon assumptions. It is perfectly possible to change these and get a different policy recommendation. That, indeed, is one of the great virtues of this sort of integrated assessment model. Climate change is added in as just another factor of production. In the Nordhaus (and Stern) world, man-made capital is a substitute for environmental capital, including the climate. There is nothing 'special' about the climate.

But it may be naive to assume that substituting for environmental systems is so easy. Feedbacks in the system may be such that as climate change unfolds, the return on capital and hence the discount rate falls. Environmental damage may slow or stop economic growth; if that were the case, we would not be much better off in the future. And if we are not so well off in growth terms, Nordhaus's slower and more measured policy approach may not be so favourable over taking rapid action now. In other words, Stern's conclusion might be correct, but not his derivation of it - right answer, wrong analysis.

Published online: 2 October 2008

doi:10.1038/climate.2008.104

\section{Dieter Helm}

Dieter Helm is professor of energy policy at the University of Oxford, UK. e-mail:dieter@dhelm.co.uk 